\title{
The Construction and Identification of Induced Pluripotent Stem Cells Derived from Acute Myelogenous Leukemia Cells
}

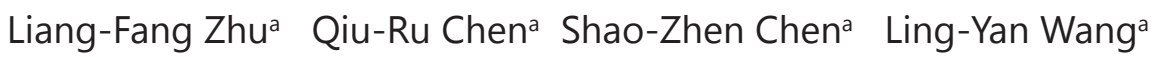 \\ Xiao-Feng Luo Jin-Hua Ren ${ }^{a}$ Xiao-Hong Yuan ${ }^{a}$ Xue-Qiong Wua Yan-Ling Zeng ${ }^{b}$ \\ Min Xiao ${ }^{a}$ Yong-Quan Chen ${ }^{a}$ Ying-Yu Chen ${ }^{a}$ Min-Hui Lin ${ }^{a}$ Zheng-Jun Wu \\ Zhi-Zhe Chen ${ }^{\mathrm{a}}$ Jian-Da Hu ${ }^{\mathrm{a}}$ Ting Yang ${ }^{\mathrm{a}}$
}

aDepartment of Hematology, Fujian Institute of Hematology, Fujian Provincial Key Laboratory of Hematology, Fujian Medical University Union Hospital, Fuzhou, 'bepartment of Hematology, Affiliated Nanping First Hospital of Fujian Medical University, Nanping, P.R. China

\section{Key Words}

Acute myelogenous leukemia $\cdot$ Induced pluripotent stem cell $\bullet$ Reprogramming $\bullet$ OCT4 $\bullet$ SOX2 - KLF4 • C-MYC • Cell pluripotent factor

\begin{abstract}
Objective: The present study aimed to establish an induced pluripotent stem cell (iPSC) line from acute myelogenous leukemia (AML) cells in vitro and identify their biological characteristics. Methods: Cells from the AML-infiltrated skin from an M6 patient were infected with a lentivirus carrying OCT4, SOX2, KLF4 and C-MYC to induce iPSCs. The characteristics of the iPSCs were confirmed by alkaline phosphatase (ALP) staining. The proliferation ability of iPSCs was detected with a CCK-8 assay. The expression of pluripotency markers was measured by immunostaining, and the expression of stem cell-related genes was detected by qRT-PCR; distortion during the induction process was detected by karyotype analysis; the differentiation potential of iPSCs was determined by embryoid body-formation and teratomaformation assays. ALP staining confirmed that these cells exhibited positive staining and had the characteristics of iPSCs. Results: The CCK-8 assay showed that the iPSCs had the ability to proliferate. Immunostaining demonstrated that iPSC clones showed positive expression of NANOG, SSEA-3, SSEA-4, TRA-1-60 and TRA-1-81. qRT-PCR results revealed that the mRNA expression of Nanog, Lin28, Cripto, FOX3, DNMT3b, DPPA2, and DPPA4 significantly increased in iPSCs. Karyotype analysis found no chromosome aberration in the iPSCs. The results of the embryoid body-formation and teratoma-formation assays indicated that the iPSCs had the potential to differentiate into all three germ layers. Conclusion: Our study provided evidence that an iPSC line derived from AML cells was successfully established.
\end{abstract}

L.-F. Zhu, Q.-R. Chen and S.-Z. Chen are regarded as co-first author.

Dr. Ting Yang

and Dr. Jian-Da Hu

KARGER
Department of Hematology, Fujian Institute of Hematology, Fujian Provincial Key

Laboratory of Hematology, Fujian Medical University Union Hospital, No. 29,

Xinquan Road, Gulou District, Fuzhou 350001, Fujian Province (P. R. China)

E-Mail yang.hopeting@gmail.com / drjiandahu@163.com 


\section{Introduction}

Acute myelogenous leukemia (AML) is a common malignant cancer of the blood system and is defined as an increase in myelogenous blasts in bone marrow resulting in inhibition of normal hematopoietic functions [1]. The mean age of AML patients is 67 years, and most AML cases occur in old age [2]. Nonetheless, AML constitutes the most common diagnostic group of childhood cancers worldwide [3]. Due to the highly heterogenous nature of AML, treatment regimen planning and prognosis prediction based on the patient's cell genetics and molecular phenotype is particularly important for AML patients [4]. With the progress of molecular biology and immunology research in recent years, the treatment of AML generally includes several layers of therapies related to cell genetics diagnosis, molecular genetics diagnosis, prognosis, and targeted and personalized treatments [5]. Therefore, it is of great significance for AML patients to explore the potential of personalized and targeted therapy in order to improve the rate of complete remission, prognosis and survival time.

Our previous study showed that leukemia cells of M4 AML patients could induce embryonic stem (ES) cell clones; however, the cell clones were highly differentiated at later passages, according to our previous study. Therefore, leukemia cells of M6 AML patients were selected to generate and characterize induced pluripotent stem cells (iPSCs) in our study. Hematopoietic stem cell transplantation has been one of the available therapies to treat AML [6]. Previous studies have demonstrated that evaluating the efficiency of both ESCs and iPSCs in cardiac cell therapy in an in vitro analysis is currently the focus of mainstream research $[7,8]$, but in recent years, iPSCs have become a great alternative for the treatment of AML [9]. These cells share many similarities with embryonic stem cells (ESCs), which serve as a model to study molecular embryogenesis and have a great potential for applications in regenerative medicine [10], including multilineage differentiation and self-renewal [11]. Moreover, embryonic stem cells (ESCs) can be differentiated into cardiac myocyte-like cells [12] Both iPSCs and ESCs are easily accessible and expandable in culture and have broad developmental potential and a high capacity for reproducibly to differentiate into spontaneously beating cardiac cells in vitro [13]. iPSCs were first discovered by Takahashi in 2006 when they used a lentiviral vector to successfully introduce OCT4, SOX2, C-MYC and KLF4 transcription factors into mouse skin fibroblasts, thus obtaining liver cells with strong self-renewal ability and pluripotency, which were subsequently named iPSCs [14]. iPSCs have been used for the successful treatment of sickle cell anemia and hemophilia A in mice $[15,16]$. A previous study has also shown that iPSCs can be efficiently induced into red blood cells [17], indicating the potential for the use of iPSCs in the clinical treatment of patients with hematological diseases. However, there are few reports on the relationship between iPSCs and $\mathrm{AML}$, and in this study, we attempted to reprogram the tissue cells from the leukemia-infiltrated skin of an AML patient into iPSCs and to analyze their biological characteristics for finding new treatment methods for personalized therapies of AML.

\section{Materials and Methods}

\section{Ethics statement}

The present study was performed in accordance with the guidelines established by the Medicine Ethics Review Committee of Fujian Institute of Hematology, Fujian Provincial Key Laboratory of Hematology, Fujian Medical University Union Hospital, and the patient signed the form of consent. Animal experiments were designed with the consent of the animal ethics committee, and all studies were conducted strictly in accordance with international standards.

Study subjects and grouping

Sample cells were from an M6 acute erythroleukemia patient (male, 21 years of age) in Fujian Institute of Hematology, Fujian Provincial Key Laboratory of Hematology, Fujian Medical University Union Hospital, which had been diagnosed by morphology, immunology, cytogenetics and molecular biology (MICM) typing. The CD13, CD33, and CD117 markers of this patient were all positive as shown by flow cytometry. The 
detection of leukemia fusion genes was negative, and the karyotype was normal. The cells were divided into the following groups: cells isolated from the infiltrated skin tissue of the M6 AML patient (leukemia-initiating cell (LIC) group) was used as the negative control group; iPSCs induced from the cells in the infiltrated skin tissue of the M6 AML patient (iPSC group) was used as the experimental group; human embryonic stem cells $\mathrm{X}-02$ (hELICs, Cellapy, Beijing, China) were used as the positive control group.

\section{Sample collection and cell culture}

Sterile surgical resection of leukemia-infiltrated skin tissue in the left inguinal region of the M6 AML patient was performed to collect a $15 \times 15 \mathrm{~mm}$ specimen, which was rinsed 2 3 times by phosphatebuffered saline (PBS) as well as Dulbecco's Minimal Essential Medium (DMEM) containing 10\% fetal bovine serum (FBS) and dual antibodies. The skin was separated from the fat tissue and cut into $1 \times 1 \mathrm{~mm}$ pieces, which were placed on the bottom of a 6-well plate. One to 2 drops of DMEM medium containing $10 \%$ FBS were added to maintain the moisture on the skin tissue. After inversion, the skin samples were placed in a $5 \% \mathrm{CO}_{2}$ incubator at $37^{\circ} \mathrm{C}$ for $6 \mathrm{~h}$. DMEM culture medium containing $10 \% \mathrm{FBS}$ but no antibodies was then added to continue the tissue culture. Every $2 \sim 3$ days, the medium was changed until the cells reached $80 \%$ confluency, after which the cells were passaged in a 1:3 ratio. The second generation of cells was collected, and a portion of the cells was used for the induction of iPSCs. The remaining cells were expanded into a certain number before being collected and cryopreserved for future use. The isolated cells were termed LICs.

\section{Induction and passage of pluripotent stem cells}

The cultured adherent LICs were inoculated in T25 cell culture flasks at $3 \times 10^{5}$ LICs per flask. At 24 $\mathrm{h}$ after inoculation, a lentivirus mixture (Genepharma, Shanghai, China) carrying OCT4, SOX2, C-MYC and KLF4 factors were used to infect and induce the cells at a target cell ratio (MOI) of 10:1. The contents of the adenovirus expression system mix are shown in Table 1 . LICs were cultured in a $5 \% \mathrm{CO}_{2}$ incubator at $37^{\circ} \mathrm{C}$ after the lentivirus mixture was added. Following $24 \mathrm{~h}$ of lentivirus infection, the supernatant was discarded carefully. LICs were digested by adding $0.25 \%$ trypsin in a $37^{\circ} \mathrm{C}$ incubator for $5 \mathrm{~min}$. An equal volume of DMEM containing 10\% FBS was added to resuspend the cells. The resuspend and induced LICs were transferred onto a layer of MEF feeder cells, with the cells in each T25 flask uniformly transferred into one 6-well plate. The DMEM medium containing $10 \%$ FBS was added, and the cells were cultured in a $5 \% \mathrm{CO}_{2}$ incubator at $37^{\circ} \mathrm{C}$. After $48 \mathrm{~h}$ of cell culture, the induced LICs were washed with $1 \mathrm{~mL}$ hydroxyethyl starches (hES) after the supernatant was removed, and then hES complete culture medium was added. The medium was changed every other day after cell clone growth was observed. After 7 days, the cell clones were gently triturated into smaller cell colonies and were transferred into a 24-well plate, which had a layer of CF- 1 feeder cells placed there 2 days before. The cells were cultured continuously using an hES medium, and the medium was changed daily until 3 generations of cells could be stably passaged. The cells were then collected for subsequent identification.

\section{Flow cytometry}

A FACSCalibur flow cytometer (Becton, Dickinson and Company, NJ, USA) was used to detect the common leukocyte antigen (CD45), the lymphoid antigens (CD2, CD3, cCD3, CD19, CD20 and HLA-D), the myelogenous antigens (CD13, CD33, CD117 and cMP0), the red blood cell antigens (CD71 and CD235a), and the stem and progenitor cell-associated antigens (CD34, CD38, CD90 and CD133). The normal cell passage was performed to collect LICs and iPSC separately, and the cells were placed in 15-mL centrifuge

Table 1. Adenovirus expression system mix. Note: MOI, multiplicity of infection; DMEM, Dulbecco's Minimal Essential Medium

\begin{tabular}{lcc}
\hline Group & Concentration & MOI \\
\hline OCT4 & $3.3 \times 10^{6} \mathrm{TU} / \mathrm{mL}$ & $0.90 \mathrm{~mL}$ \\
SOX2 & $2.8 \times 10^{6} \mathrm{TU} / \mathrm{mL}$ & $0.11 \mathrm{~mL}$ \\
C-MYC & $4.3 \times 10^{6} \mathrm{TU} / \mathrm{mL}$ & $0.70 \mathrm{~mL}$ \\
KLF4 & $2.3 \times 10^{6} \mathrm{TU} / \mathrm{mL}$ & $1.30 \mathrm{~mL}$ \\
Polybrene & $10 \mathrm{mg} / \mathrm{mL}$ & $6 \mu \mathrm{L}$ \\
DMEM medium & Containing $10 \%$ FBS & $2 \mathrm{~mL}$ \\
\hline
\end{tabular}


tubes. The iPSC clones underwent 3 cycles of natural sedimentation, and the supernatant was discarded to remove most of the components of the CF- 1 feeder cells mixed in the iPSC clones. CD3 ${ }^{+}$cells underwent centrifugation at $1000 \mathrm{rpm}$ for $5 \mathrm{~min}$, and the supernatant was discarded. A volume of $5 \mathrm{~mL}$ PBS was added to resuspend the cells, and the cell concentration was determined by counting the cells. In each $1.5-\mathrm{mL}$ EP tube, $1 \times 10^{5}$ cells were added and centrifuged at $1000 \mathrm{rpm}$ for $5 \mathrm{~min}$, followed by discarding of the supernatant. In each EP tube, $0.3 \mathrm{~mL}$ of PBS was added to resuspend the cells. According to the operation instructions of the reagent, $1 \mu \mathrm{L}$ of CD3 monoclonal antibody was added into 1953 cells, and $1 \mu \mathrm{l}$ of CD34 monoclonal antibody was added into HL60 cells. After mixing, the cells were placed at $4{ }^{\circ} \mathrm{C}$ and incubated for $30 \mathrm{~min}$. The cells were washed with PBS solution 2 times, and then $400 \mu \mathrm{l}$ of PBS solution was used to resuspend the cells for flow cytometry detection.

\section{Alkaline phosphatase (ALP) staining}

iPSCs were cultured in 12-well plates to form typical iPSC clones, followed by the detection of characteristics of stem cells by alkaline phosphatase (ALP) staining and the visualization of the corresponding images. The dying procedures and methods were in accordance with the instructions for the ALP Staining Kit (Millipore, USA).

\section{Cell proliferation assay}

Each generation of cells was digested, counted, and inoculated in 96-well plates and diluted to a concentration of $2 \times 10^{3}$ cells $/ \mathrm{ml}$. The cells were plated in three parallel wells and underwent conventional culture. A Cell Counting Kit-8 (DOJINDO, Japan) was used to assay cell proliferation, and the growth curve was plotted. The specific steps were in strict accordance with the instructions for the kit.

\section{Detection of pluripotency marker expression}

LICs, iPSC and X-02 were seeded, separately, in 24-well plates with each well receiving 10 to 20 clones. After culturing for 3 days, the supernatant was removed, and the cells were washed 2 times with PBS and fixed with 4\% PFA for 20 min at room temperature. After washing 2 times with PBS, the cells were permeated with PBS containing 0.5\% Triton X-100 for 15 min and blocked with PBS containing 2\% BSA + $5 \%$ normal goat serum for $1 \mathrm{~h}$ at room temperature. The cells were then incubated with primary antibodies including NANOG, SSEA-3, SSEA-4, TRA-1-60 and TRA-1-81 (all were purchased from Abcam, USA) at $4^{\circ} \mathrm{C}$ overnight. On the next day, the cells were washed 3 times with PBS, and secondary antibodies, Alexa Fluor ${ }^{\circledR}$ 555 R goat anti rabbit IgG, Alexa Fluor ${ }^{\circledR} 555$ goat anti-mouse IgG, and Alexa Fluor ${ }^{\circledR} 555$ goat anti-mouse IgM (Invitrogen, USA), were added and incubated at room temperature for $1 \mathrm{~h}$. Subsequently, the cells were washed with PBS 3 times, stained with $1 \mu \mathrm{g} / \mathrm{ml}$ DAPI for $5 \mathrm{~min}$, washed 3 times again with PBS, mounted, observed and imaged under a fluorescence microscope.

\section{Detection of stem cell-related genes}

A portion of the cells in each group were collected and lysed with Trizol (Invitrogen, USA). The RNA was extracted, and the cDNA was synthesized by a reverse transcription kit (Tiangen Biotech Co., Ltd, Beijing, China). The quantitative real-time PCR (qRT-PCR) method was used to detect the expression of stem cell-related genes. The specific procedures were in accordance with the instructions for the SYBR Green PCR Master Mix reagent (Invitrogen, USA). The primer sequences of the stem cell-related genes are shown in Table 2.

Analysis of chromosome karyotype in induced pluripotent cells

iPSCs were inoculated in T25 flasks according to a normal passage method. After $72 \mathrm{~h}, 0.05 \mathrm{~mL}$ colchicine $(25 \mu \mathrm{g} / \mathrm{mL})$ was added and gently mixed, and the cells were cultured for $4 \mathrm{~h}$. The cells were then collected and centrifuged for $5 \mathrm{~min}$ at $1000 \mathrm{rpm}$. The supernatant was discarded, and $4 \mathrm{ml}$ of $0.4 \%$ sodium citrate and $4 \mathrm{~mL}$ of $0.4 \% \mathrm{KCl}$ solution preheated at $37^{\circ} \mathrm{C}$ were added to the cells. After gentle mixing, the mixture was incubated at $37^{\circ} \mathrm{C}$ for $5 \mathrm{~min}$. Then, pre-cooled and newly prepared fixing liquid (methanol: acetic acid ratio was 3: 1) was used to fix the cells 3 times, with each time taking $30 \mathrm{~min}$. Subsequently, 2 $\mathrm{mL}$ of pre-cooled fixing liquid was added and mixed gently. From a height of $50 \mathrm{~cm}$, the cell suspension was dropped onto slides already cleaned by soaking in $4-6^{\circ} \mathrm{C}$ ice water, promoting the cells to spread on the 
Table 2. Primer sequences of stem cell-related genes for qRT-PCR. Note: qRT-PCR, quantitative real-time polymerase chain reaction

\begin{tabular}{lll}
\hline Gene & & Primer sequence \\
\hline Nanog & sense & 5'-ATGGAGGGTGGAGTATGGTTGG -3' \\
& antisense & 5'-AGGCTGAGGCAGGAGAATGG -3' \\
Lin28 & sense & 5'- CAGGTGCTACAACTGTGGAGG -3' \\
& antisense & 5'- GCACCCTATTCCCACTTTCTCC -3' \\
FoXD3 & sense & 5'- TGGGCTTTCAGGTTATTTGACT-3' \\
& antisense & 5'-AAGCCAAGAACAGCCTAGTGA-3' \\
Cripto & sense & 5'- TACCTGGCCTTCAGAGATGACA-3' \\
& antisense & 5'-CCAGCATTTACACAGGGAACAC -3' \\
DPPA4 & sense & 5'-GGAGCCGCCTGCCCTGGAAAATTC-3' \\
& antisense & 5'-GGAGCCGCCTGCCCTGGAAAATTC-3' \\
DPPA2 & sense & 5'- CCGTCCCCGCAATCTCCTTCCATC -3' \\
& antisense & 5'-ATGATGCCAACATGGCTCCCGGTG-3' \\
DNMT3B & sense & 5'-TCAAAGTTTCTGCTGCTCACAGGG-3' \\
& antisense & 5'- TTTTTCCTGATATTCTATTCCCAT -3' \\
G3PDH & sense & 5'-AGGTCGGAGTCAACGGATTTGG-3' \\
& antisense & 5'-AGGCTGTTGTCATACTTCTCATGG-3' \\
\hline
\end{tabular}

Table 3. Primer sequences of

\begin{tabular}{|c|c|c|c|}
\hline Germ layer & Gene & & Primer sequence \\
\hline \multirow[t]{4}{*}{ Endodermal layer } & AFP & sense & 5'- TCGTTGCTTACACAAAGAAAGCCC-3' \\
\hline & & antisense & 5'- CCGATAATAATGTCAGCCGCTCCC -3' \\
\hline & TNNT2 & sense & 5'- 'TTCACCAAAGATCTGCTCCTCGCT -3' \\
\hline & & antisense & 5'- TTATTACTGGTGTGGAGTGGGTGTGG -3 \\
\hline \multirow[t]{4}{*}{ Mesodermal layer } & ACTC1 & sense & 5'- ACCACAGGCATTGTTCTGGACTC - -3' \\
\hline & & antisense & 5'- AATCCAGGGCGACATAGCACAG -3' \\
\hline & Brachyury & sense & 5'- СCCGTCTCCTTCAGCAAAGTC -3' \\
\hline & & antisense & 5'- TAAGAGCTGTGATCTCCTCGTTCTG -3' \\
\hline \multirow[t]{4}{*}{ Ectodermal layer } & Nestin & sense & 5'- СTTCCCTCAGCTTTCAGGACCC -3' \\
\hline & & antisense & 5'- TTCCTCCCACCCTGTGTCTG -3' \\
\hline & PAX6 & sense & 5'- ATGGCGAATAATCTGCCTATGC - -3' \\
\hline & & antisense & 5'- CCAGGGGAAATGAGTCCTGTG - -3' \\
\hline \multirow{2}{*}{$\begin{array}{l}\text { Internal } \\
\text { reference }\end{array}$} & GAPDH & sense & 5'- AGGTCGGAGTCAACGGATTTGG - $3^{\prime}$ \\
\hline & & antisense & 5'- AGGCTGTTGTCATACTTCTCATGG -3' \\
\hline
\end{tabular}

slides. The slides were then immediately baked in a $80^{\circ} \mathrm{C}$ constant-temperature drying oven for $15 \mathrm{~min}$. After Giemsa staining, at least 20 mitotic images were analyzed.

\section{Differentiation of three germ layers in embryoid bodies}

iPSCs obtained from LICs were propagated and digested using normal cell passage procedures and made into small clumps. They were then cultured in a suspension. Three days later, the medium was changed for the first time. Subsequently, the culture media was changed every 2-3 days, and the cells were evaluated with a microscope and imaged. Embryoid bodies (EB) were collected at 7 days and 14 days and were lysed by Trizol. RNA was extracted, and cDNA was made. The expression of markers relevant to different germ layers was detected by qRT-PCR. The primer sequences of the marker genes for the three germ layers are shown in Table 3.

\section{Detection of teratoblastoma formation}

iPSCs cultured for more than 10 generations were digested and cleaned according to a normal passage method. After removal of feeder cells, the iPSCs were suspended in $400 \mu \mathrm{L}$ of culture medium and injected into NOD-SCID mice (Shanghai SLAC Laboratory Animal Co., Ltd, Shanghai, China) subcutaneously in two hind legs. Each injection point received $4 \times 10^{6}$ cells. The nude mice were injected with either LICs or hELICs cells as controls. Four weeks later and after the teratoblastoma grew to a diameter of 1 to $2 \mathrm{~cm}$, the nude mice were sacrificed, and the teratoblastoma was removed by surgical dissection. The tumor tissue was fixed with $4 \%$ paraformaldehyde overnight, embedded in paraffin, sectioned, stained with $\mathrm{HE}$, and observed under the microscope. Different types of tissue structures were photographed. 


\section{Cellular Physiology Cell Physiol Biochem 2017;41:1661-1674

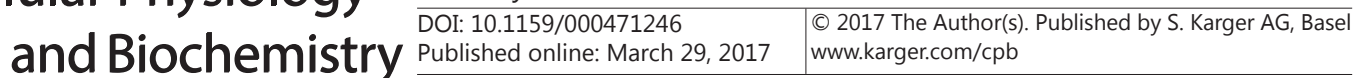 Zhu et al.: AML and iPSCs}

Statistical analysis

Data were analyzed by SPSS 21.0 statistical software. All data were presented as the mean \pm standard deviation. The differences among groups were compared using single-factor analysis of variance. The comparisons between two groups were verified with the Newman-Keuls test. A $P$ value of $<0.05$ was considered significant.

\section{Results}

The iPSC clones and passage

The status of the iPSC clones was optimized during passage. After cells were cultured for three generations, the borders of the iPSCs were distinct; the cells were arranged tightly, and the karyoplasmic ratio increased. All of the above morphological features were similar to those of embryonic stem (ES) cells (Fig. 1).

Detection of the immune phenotypes of iPSCs

The flow cytometry results showed that $97.88 \%$ of the LICs expressed the myelogenous antigen CD13, 76.27\% expressed the red blood cell-specific antigen CD71, and 10.76\% expressed CD235a; the expression of CD13, CD71 and CD235a in the generated iPSCs was all negative. For CD45, the expression in LICs was $90.24 \%$, while the expression decreased to $9.45 \%$ of the iPSCs. However, more than $90 \%$ of both the LICs and IPSCs expressed CD90 (Fig. 2). These results indicate that in the course of the transformation of LICs to iPSCs, the expression of antigen markers specific to the immune phenotypes of LICs tended to decrease or disappear, and the previously non-expressed stem cell-specific CD antigens were expressed.

Comparisons of the cell activity and proliferation in the cells among the three groups

ALP staining showed that the selected iPSC-like cell clones and the positive control $\mathrm{X}-02$ cells were positive, but the LICs in the negative control group were negative. These preliminary results showed that the iPSCs had the characteristics of stem cells (Fig. 3). The cell growth curve of each group showed that iPSCs and X-02 cells in the positive control group had stronger proliferation ability than the LIC group (Fig. 4).

Comparison of the NANOG, SSEA-3, SSEA-4, TRA-1-60 and TRA-1-81 expression among the three groups

Immunostaining showed that in the iPSCs and positive control X-02 cells, the expression of pluripotency markers NANOG, SSEA-3, SSEA-4, TRA-1-60 and TRA-1-81 were all positive, but the expression of these markers was all negative in the negative control LICs (Fig. 5).

Fig. 1. The morphological features of iPSC clones after passages under a fluorescence microscope $(\times 400)$. Note: $A$, the morphological features of iPSC clones under a fluorescence microscope after culturing for one generation; $\mathrm{B}$, the morphological features of iPSC clones under a fluorescence microscope after culturing for three generations; iPSC, induced pluripotent stem cell.

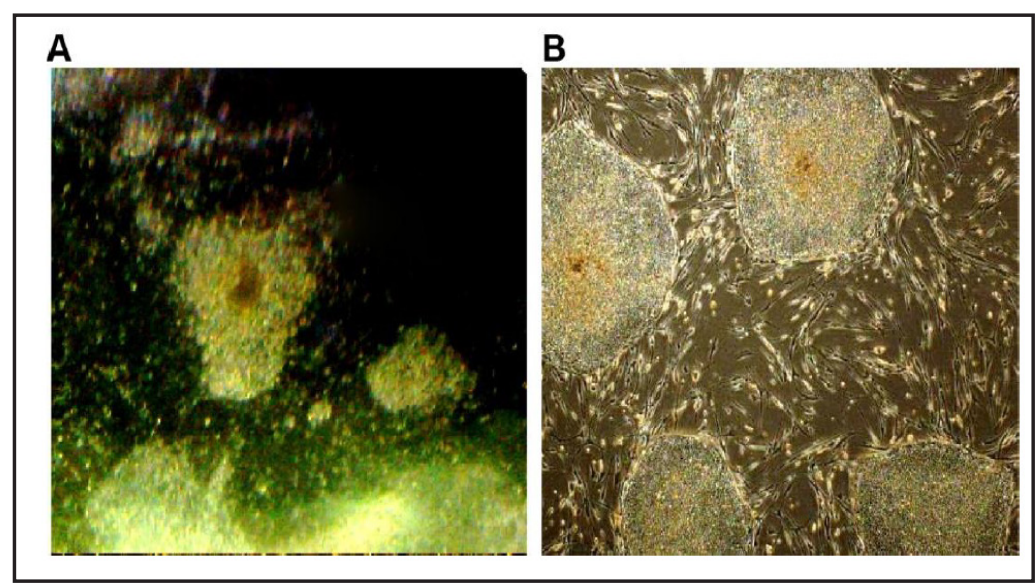


Fig. 2. Flow cytometry for the detection of the immune phenotypes of the cells. Note: A, LICs; B, iPSCs; LICs, leukemia-initiating cells; iPSCs, induced pluripotent stem cells.

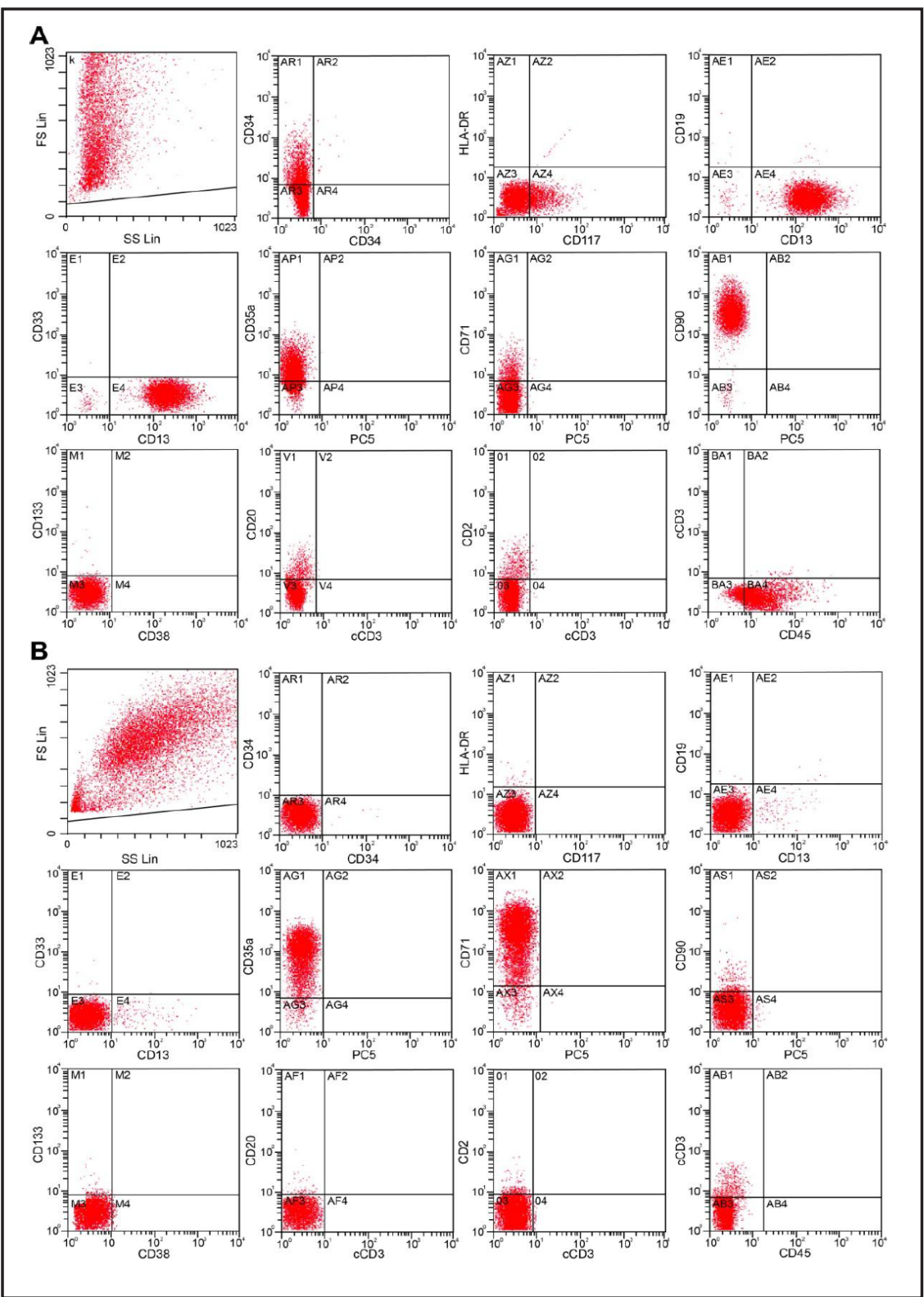

Fig. 3. Comparison of the alkaline phosphatase staining results of LICs, iPSCs and X-02 cells $(\times 400)$. Note: A, the LIC group; $B$. the iPSC group; $C$, the $\mathrm{X}-02$ group; LIC, leukemia-initiating cell; iPSC, induced pluripotent stem cell.
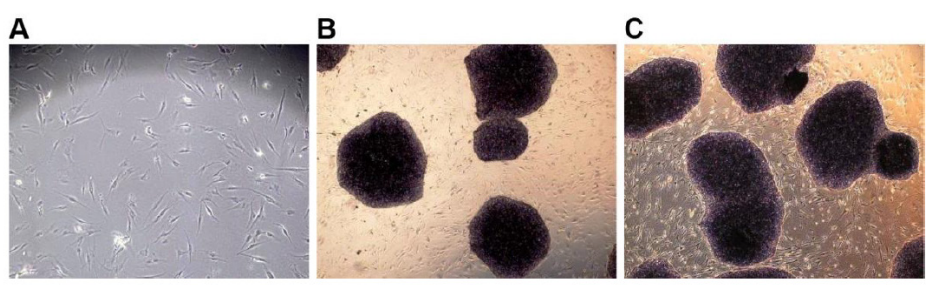

Comparison of the Nanog, Lin28, Cripto, FOX3, DNMT3b, DPPA2 and DPPA4 expression among the stem cells in the three groups

The qRT-PCR results showed that the expression of stem cell-associated genes Nanog, Lin28, Cripto, FOX3, DNMT3b, DPPA2 and DPPA4 was significantly higher in iPSCs than that observed in the negative control LICs (all $P<0.01$ ); there were no significant differences between the positive control X-02 cells and iPSCs (all $P>0.05$ ) (Fig. 6). 
Fig. 4. Cell growth curve of X-02 cells, iPSCs and LICs. Note: iPSCs, induced pluripotent stem cells; LICs, leukemia-initiating cells.

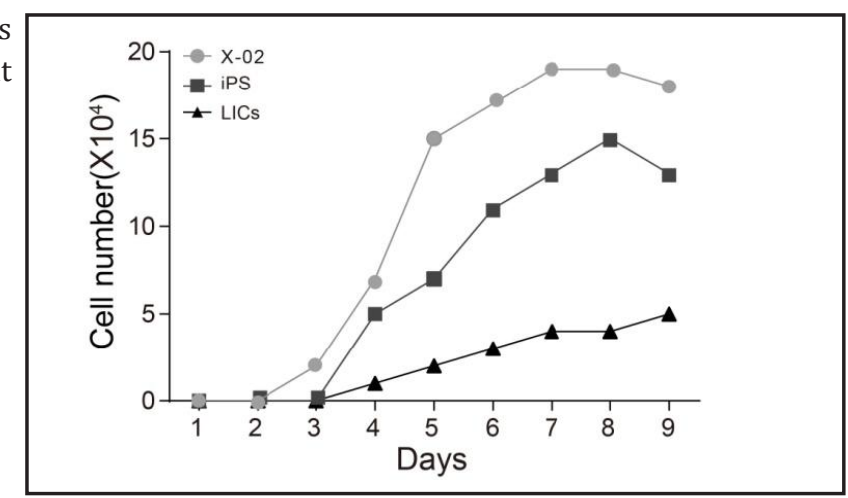

Fig. 5. Comparison of the expression of pluripotency markers NANOG, SSEA3, SSEA-4, TRA-160 and TRA-1-81 in LICs, iPSCs and X-02 cells by immunofluorescence detection (×400). Note: the magnification was $400 \mathrm{x}$; LICs, leukemia-initiating cells; iPSCs, induced pluripotent stem cells.

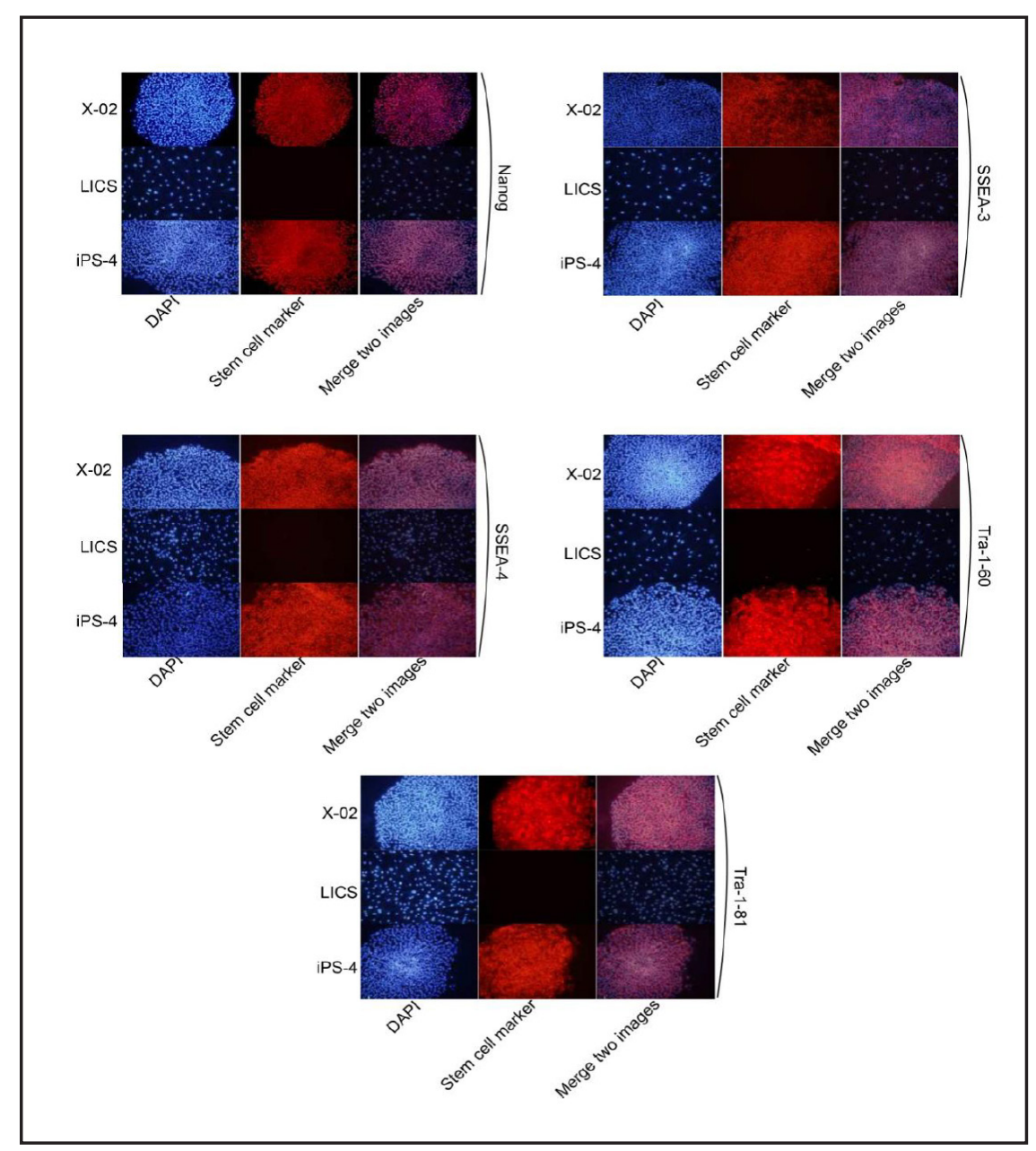

Chromosome karyotype analysis of iPSCs

The results of the karyotype analysis showed that in more than 10 generations of cell culture, all iPSCs showed a normal karyotype (Fig. 7), indicating that there was no chromosomal aberration when LICs were induced into iPSCs.

Comparison of the TNNT2, AFP, ACTC1, Brachyury, Nestin and PAX6 expression in the embryoid formations among the three groups

The iPSCs can form EBs (Fig. 8). The qRT-PCR results showed that, after the formation of EBs and compared with that before the differentiation of iPSCs, the expressions of TNNT2, AFP, ACTC1, Brachyury, Nestin and PAX6, which represent genes of the different germ layers, were all significantly up-regulated (all $P<0.01$ ) (Fig. 9). 
Fig. 6. Comparison of the expression of stem cell-related genes Nanog, Lin28, Cripto, FOX3, DNMT3b, DPPA2 and DPPA4 in LICs, iPSCs and X-02 cells by qRT-PCR detection. Note: ${ }^{* *}, P<0.01$ compared to the LIC group; LIC, leukemia-initiating cell; iPSCs, induced pluripotent stem cells; qRT-PCR, quantitative real-time polymerase chain reaction.
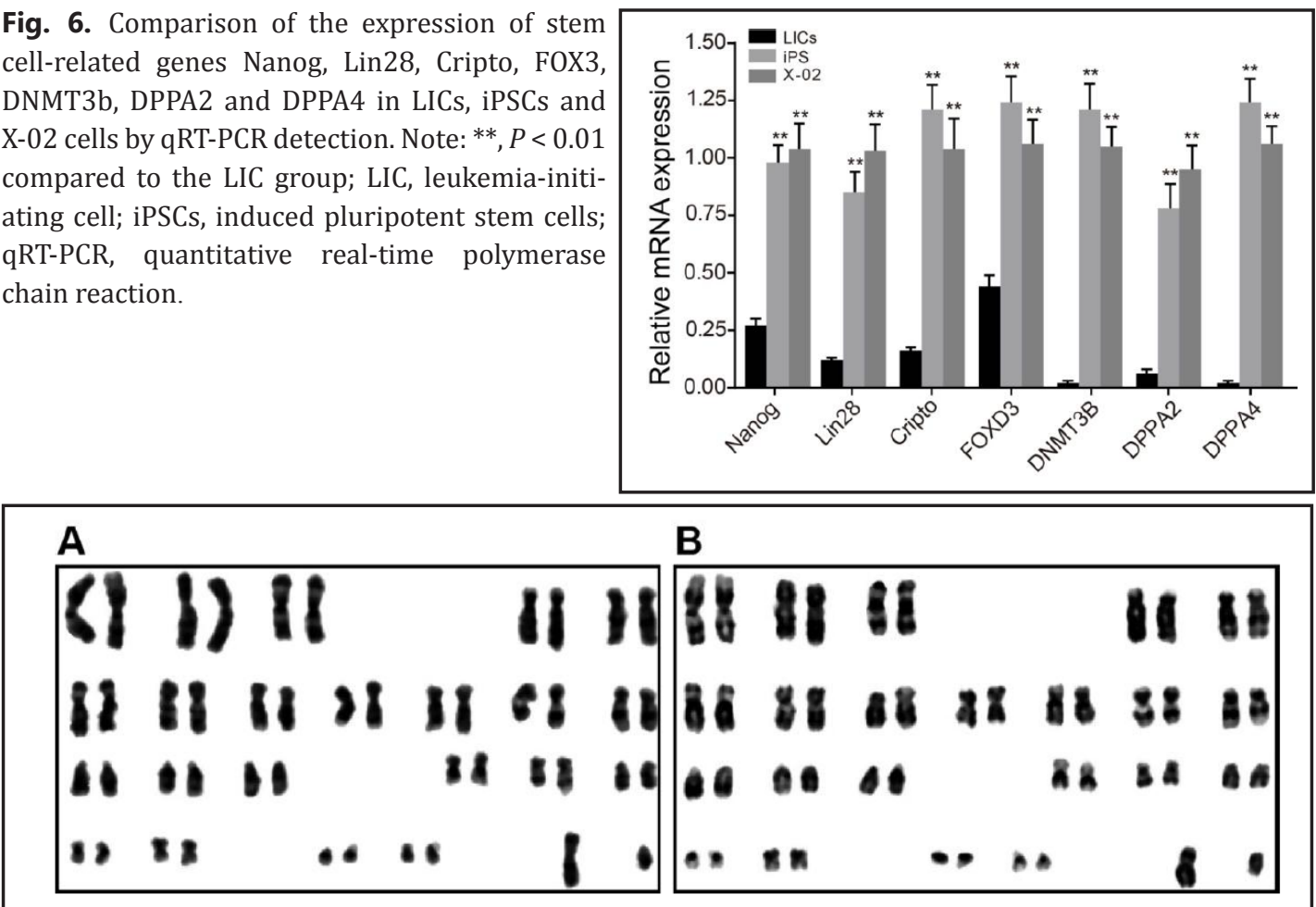

Fig. 7. Karyotype analysis of cells. Note: A, chromosome karyotype of LICs; B, chromosome karyotype of iPSCs; iPSCs, induced pluripotent stem cells.

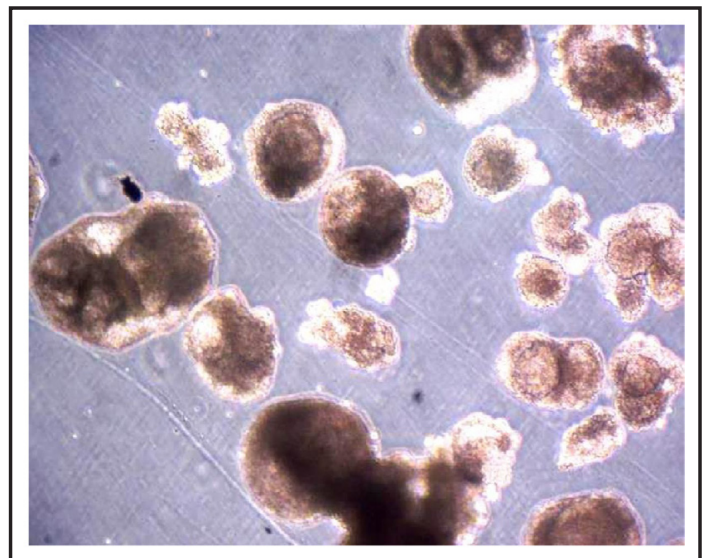

Fig. 8. The morphology of embryoid bodies formed by iPSCs $(\times 400)$. Note: iPSCs, induced pluripotent stem cells.

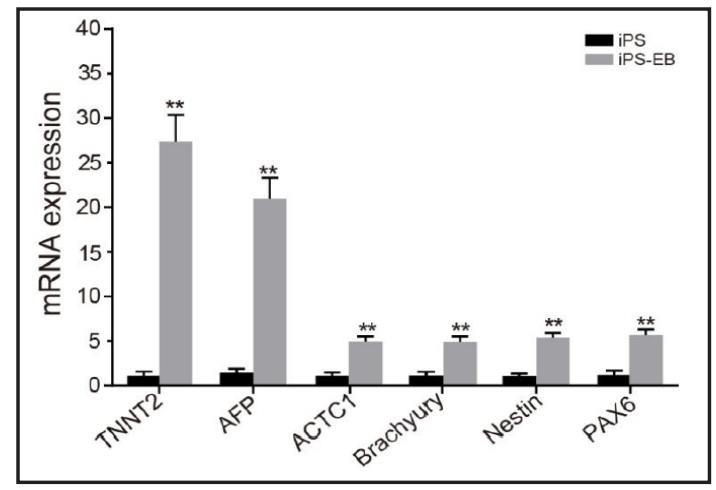

Fig. 9. Comparison of the expression of mesodermal layer-related genes TNNT2, AFP, ACTC1, Brachyury, Nestin and PAX6 in iPSCs. Note: ${ }^{* *}, P<0.01$ compared with the iPSC group; iPSC, induced pluripotent stem cell.

The differentiation function of iPSCs in vivo

Four weeks after iPSCs were injected into the root muscle in the hind legs of NOD-SCID mice, tumors with a diameter of approximately $1.5 \mathrm{~cm}$ could be seen (Fig. 10). From the tissue section of the teratoma and HE staining in the iPSC group, the teratoma was found to include tissue structures of three germ layers, including nerve tissue, bone, cartilage, intestine and respiratory tract tissue (Fig. 11). This indicates that the iPSCs have the ability to differentiate into various germ layers. 


\section{Cellular Physiology \begin{tabular}{ll|l} 
DOI: 10.1159/000471246 & $\begin{array}{l}\text { O 2017 The Author(s). Published by S. Karger AG, Basel } \\
\text { www.karger.com/cpb }\end{array}$ \\
\cline { 2 - 3 }
\end{tabular}

Fig. 10. Tumor-bearing mouse and the removed teratoma. Note: A, a tumor-bearing mouse injected with iPSCs; B, teratoma of the tumor-bearing mice injected with iPSCs; iPSCs, induced pluripotent stem cells.

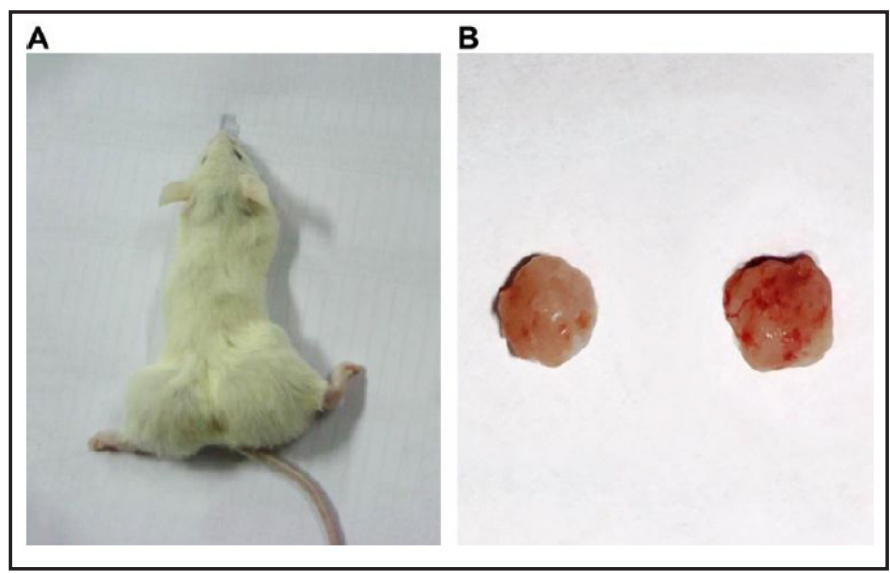

Fig. 11. HE staining of 3 germ layers formed by iPSCs. Note: A, different germ layer tissues in the teratoblastoma; $B$, neural epithelium tissue in the ectoderm layer; $\mathrm{C}$, bone tissue in the mesoderm layer; D, cartilage tissue in the mesoderm layer; E, intestinal and respiratory tissue in the endoderm layer; the magnification in $\mathrm{A}, \mathrm{B}-1, \mathrm{C}-1$, D-1 and E-1 was $40 \times$; the magnification in B-2, C-2, D-2 and E-2 was 100x; iPSCs, induced pluripotent stem cells.

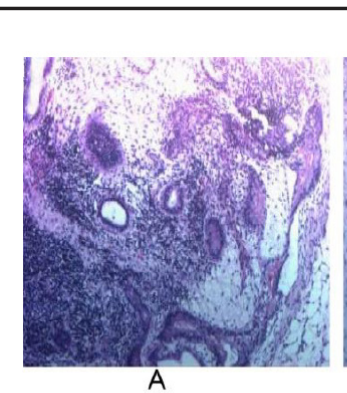

A

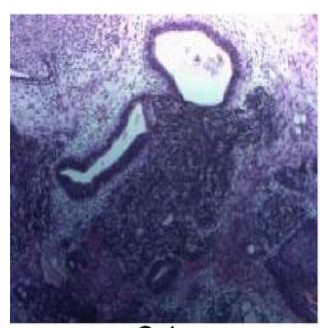

C-1

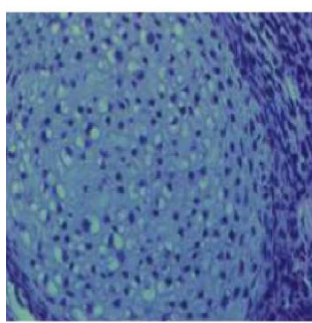

D-2

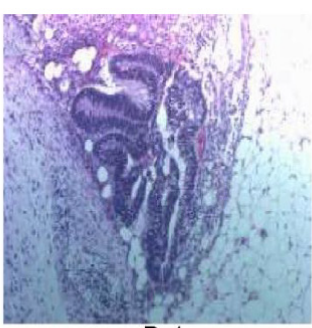

B-1
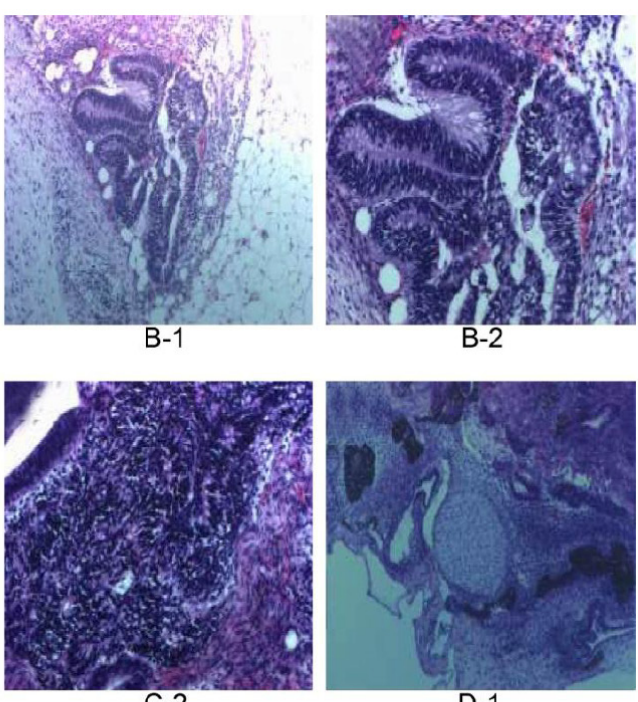

C-2

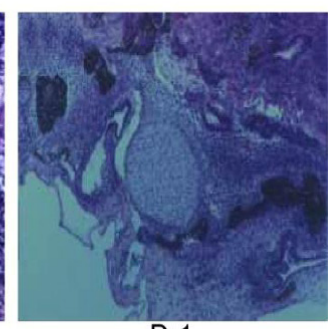

D-1

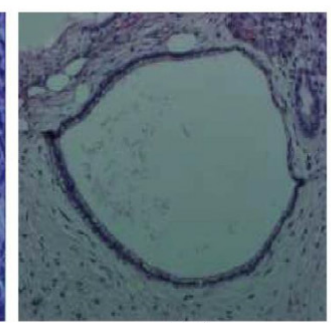

E-1

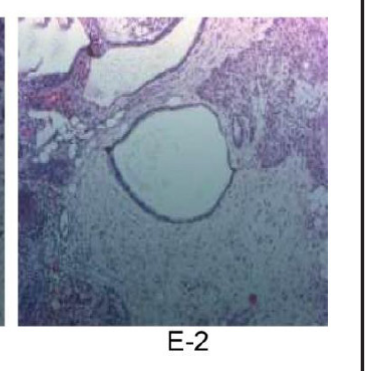

\section{Discussion}

In this study, lentivirus vectors carrying SOX2, KLF4, C-MYC4 and OCT4 were used to infect leukemia cells from the infiltrated skin of an M6 AML patient to induce the formation of iPSCs. Ultimately, our study confirmed that the induction of iPSCs was successful, and the generated iPSCs had the characteristics of pluripotent stem cells that have the potential to differentiate into the three germ layers.

In the personalized treatment plan of AML, hematopoietic stem cell transplantation is currently the main treatment method for AML, and it will become an important AML therapy with the progression of AML research [6]. The iPSCs are a kind of induced pluripotent stem cell that were initially obtained by inducing mouse skin fibroblasts, and they have a strong -renewal and differentiation ability [14]. In the present study, the expression of CD13, CD71 
and CD235a in iPSCs was found to be negative, and the expression of CD45 was decreased. CD13 is a myelogenous antigen commonly expressed in leukemia [18]. CD71 and CD235a are red blood cell antigens expressed on the surface of leukemia cells $[19,20]$. CD45 is also known as the common leukocyte antigen and is a specific glycoprotein of white blood cells [21]. Moreover, a previous study also confirmed that the expression of CD45 is missing in the successful extraction of iPSC-MSCs [22]. Therefore, the results indicate that the expression of antigen markers for an LIC-specific immune phenotype is reduced or disappeared during the transformation of LICs to iPSCs.

In addition, this study showed that compared with that of the LICs, the iPSCs have a stronger proliferation ability that is similar to that of X-02 cells. ESCs have been widely regarded as one of the promising cell sources that are used not only for cell replacement therapy butalso as the best platform for developmental research, screening and drug discovery [23]. Moreover, a previous study has demonstrated that iPSCs possess the characteristics of embryonic stem cells [24], and embryonic stem cells have also been demonstrated to have an unlimited proliferative potential as well as the ability to differentiate into almost any type of tissue [25]. Because of this, iPSCs have a stronger ability to proliferate than differentiated cells and were predicted to be transferred into adult cells via parallel mathematical modeling and numerical simulation [26]. Studies have shown that in successfully induced stem cells with a differentiation potential, a number of embryonic stem cell protein markers such as NANOG, SSEA-3, SSEA-4, TRA-1-60 and TRA-1-81 are expressed [27-29], wherein SSEA-3 and SSEA-4 are cell membrane proteins, TRA-1-60 and TRA-1-81 are cytoplasmic proteins, and NANOG is a cell nuclear protein [30-32]. The iPSCs obtained in this study also expressed the above proteins, indicating that the iPSCs obtained in this study were similar to embryonic stem cells.

Finally, the iPSCs obtained in this study showed a significant up-regulation of Nanog, Lin28, Cripto, FOX3, DNMT3b, DPPA2 and DPPA4. Nanog is a regulatory factor that arrests the embryonic stem cells in an undifferentiated state and plays a key role in maintaining the subpluripotency of stem cells [33]. Lin28 plays an important role in the growth and metabolism of stem cells [34]. Cripto plays an important part in maintaining the undifferentiated state of stem cells but is also a key gene in the early development of embryos [35]. DNMT3b is a type of methyltransferase that mediates DNA methylation and plays an important role in the growth and development of embryonic stem cells [36]. DPPA2 and DPPA4 are both recently identified specific marker genes in embryonic stem cells, wherein DPPA2 plays an important role in maintaining the undifferentiated state and proliferation of embryonic stem cells, and DPPA4 is involved in regulating the differentiation of embryonic stem cells into ectodermal cells $[37,38]$. Therefore, because of the important roles played by the above genes in the differentiation and pluripotency of adult stem cells, we can speculate that the iPSCs exhibit the characteristics of stem cells in all respects. The characteristics of iPSCs tend to be similar to those of embryonic stem cells in that the stem cell-related regulatory factors are also significantly up-regulated. In addition, during the spontaneous formation of EBs by iPSCs in vitro, the cell and gene expression phenotypes of the EBs changed. In this study, after the iPSCs formed EBs in vitro, the expression of genes representative of three germ layers [39], such as TNNT2 and AFP (endodermal gene), ACTC1 and Brachyury (mesodermal gene), and Nestin and PAX6 (ectodermal gene), were all significantly up-regulated, and elevation of TNNT2 and AFP gene expression was higher than that of the genes for the other two layers. These results indicate that the iPSCs have the ability to differentiate into various germ layers, especially the inner germ layer.

This study proves that by infecting leukemia cells obtained from the infiltrated skin of an M6 AML patient using lentivirus vectors carrying OCT4, SOX2, KLF4 and C-MYC factors, iPSCs can be induced and generated, and the generated iPSCs have the characteristics and proliferation potential of stem cells and express stem cell-specific proteins and genes. This study also confirms the potential for pluripotent differentiation and safety of the obtained iPSCs. This study provided the theoretical basis for the personalized treatment of AML and may provide a technical platform for the personalized treatment of AML patients. However,

\section{KARGER}




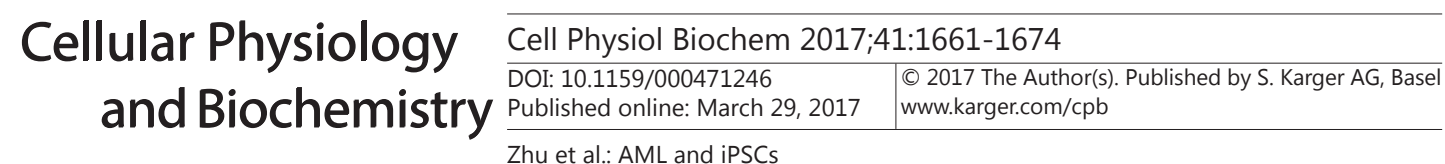

the induction efficiency and the mechanism by which the transcription factors induce leukemia cells to become iPSCs are still not clear and require further research.

\section{Acknowledgements}

We thank Prof. Lei Xiao (College of Animals, Zhejiang University, Hangzhou, Zhejiang310058, China) and Doctor Zhao Wu (Shanghai SiDanSai Biotechnology Limited Company, Shanghai 201210, China) for their scientific supports. This work was supported in part by the National and Fujian Provincial Key Clinical Specialty Discipline Construction Program, China, National High Technology Research and Development Program of China, 863 Program (2012AA02A505), National Public Health Grand Research Foundation (201202017), National Natural Science Foundation of China (81570162), Fujian Provincial Key Laboratory Foundation of Hematology (2009J1004), Natural Science Foundation of Fujian Province (2013Y0044,2013J01310) and the Backbone Talents Training Project of the Fujian Bureau of Public Health, P.R.C. (2014-ZQN-ZD-8). We would like to give our sincere appreciation to the reviewers for their helpful comments on this article.

\section{Disclosure Statement}

The authors declare that they have no competing interests.

\section{References}

1 Motyckova G, Stone RM: The role of molecular tests in acute myelogenous leukemia treatment decisions. Curr Hematol Malig Rep 2010;5:109-117.

2 Ayatollahi H, Shajiee A, Sadeghian MH, Sheikhi M, Yazdandoust E, Ghazanfarpour M: Prognostic importance of c-kit mutations in core binding factor acute myeloid leukemia: A systematic review. Hematol Oncol Stem Cell Ther DOI:10.1016/j.hemonc.2016.08.005.

-3 Boomsma F, Meerwaldt JD, Man in't Veld AJ, Hovestadt A, Schalekamp MA: Treatment of idiopathic parkinsonism with L-dopa in the absence and presence of decarboxylase inhibitors: effects on plasma levels of L-dopa, dopa decarboxylase, catecholamines and 3-O-methyl-dopa. J Neurol 1989;236:223-230.

4 Mrozek K, Marcucci G, Nicolet D, Maharry KS, Becker H, Whitman SP, Metzeler KH, Schwind S, Wu YZ, Kohlschmidt J, Pettenati MJ, Heerema NA, Block AW, Patil SR, Baer MR, Kolitz JE, Moore JO, Carroll AJ, Stone RM, Larson RA, Bloomfield CD: Prognostic significance of the European LeukemiaNet standardized system for reporting cytogenetic and molecular alterations in adults with acute myeloid leukemia. J Clin Oncol 2012;30:4515-4523.

5 Gao L, Sun J, Liu F, Zhang H, Ma Y: Higher expression levels of the HOXA9 gene, closely associated with MLL-PTD and EZH2 mutations, predict inferior outcome in acute myeloid leukemia. Onco Targets Ther 2016;9:711-722.

6 Mo XD, Zhang XH, Xu LP, Wang Y, Yan CH, Chen H, Chen YH, Han W, Wang FR, Wang JZ, Liu KY, Huang XJ: Unmanipulated Haploidentical Hematopoietic Stem Cell Transplantation in First Complete Remission Can Abrogate the Poor Outcomes of Children with Acute Myeloid Leukemia Resistant to the First Course of Induction Chemotherapy. Biol Blood Marrow Transplant 2016;22:2235-2242.

7 Xu R, Srinivasan SP, Sureshkumar P, Nembo EN, Schafer C, Semmler J, Matzkies M, Albrechtsen M, Hescheler J, Nguemo F: Effects of synthetic neural adhesion molecule mimetic peptides and related proteins on the cardiomyogenic differentiation of mouse embryonic stem cells. Cell Physiol Biochem 2015;35:2437-2450.

8 Li R, Cheng S, Wang Z: Circadian clock gene plays a key role on ovarian cycle and spontaneous abortion. Cell Physiol Biochem 2015;37:911-920.

> Nishigori K, Temma T, Onoe S, Sampei S, Kimura I, Ono M, Saji H: Development of a radioiodinated triazolopyrimidine probe for nuclear medical imaging of fatty acid binding protein 4. PLoS One 2014;9:e94668. 


\section{Cellular Physiology Cell Physiol Biochem 2017;41:1661-1674

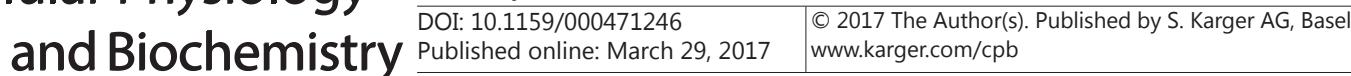

10 Wang J, Masika J, Zhou J, Wang J, Zhu M, Luo H, Hu X, Zhang L, Tang M, Gao L, Hescheler J, Liang H: Traditional Chinese medicine baicalin suppresses mESCs proliferation through inhibition of miR-294 expression. Cell Physiol Biochem 2015;35:1868-1876.

11 Yan Y, Yin P, Gong H, Xue Y, Zhang G, Fang B, Chen Z, Li Y, Yang C, Huang Z, Yang X, Ge J, Zou Y: Nucleosome Assembly Protein 1-Like 1 (Nap1l1) Regulates the Proliferation of Murine Induced Pluripotent Stem Cells. Cell Physiol Biochem 2016;38:340-350.

12 Hannes T, Wolff M, Doss MX, Pfannkuche K, Haustein M, Muller-Ehmsen J, Sachinidis A, Hescheler J, Khalil M, Halbach M: Electrophysiological characteristics of embryonic stem cell-derived cardiomyocytes are cell line-dependent. Cell Physiol Biochem 2015;35:305-314.

-13 Ivanyuk D, Budash G, Zheng Y, Gaspar JA, Chaudhari U, Fatima A, Bahmanpour S, Grin VK, Popandopulo AG, Sachinidis A, Hescheler J, Saric T: Ascorbic Acid-Induced Cardiac Differentiation of Murine Pluripotent Stem Cells: Transcriptional Profiling and Effect of a Small Molecule Synergist of Wnt/beta-Catenin Signaling Pathway. Cell Physiol Biochem 2015;36:810-830.

14 Takahashi K, Yamanaka S: Induction of pluripotent stem cells from mouse embryonic and adult fibroblast cultures by defined factors. Cell 2006;126:663-676.

15 Hanna J, Wernig M, Markoulaki S, Sun CW, Meissner A, Cassady JP, Beard C, Brambrink T, Wu LC, Townes TM, Jaenisch R: Treatment of sickle cell anemia mouse model with iPS cells generated from autologous skin. Science 2007;318:1920-1923.

16 Xu D, Alipio Z, Fink LM, Adcock DM, Yang J, Ward DC, Ma Y: Phenotypic correction of murine hemophilia A using an iPS cell-based therapy. Proc Natl Acad Sci U S A 2009;106:808-813.

17 Lu SJ, Feng Q Park JS, Vida L, Lee BS, Strausbauch M, Wettstein PJ, Honig GR, Lanza R: Biologic properties and enucleation of red blood cells from human embryonic stem cells. Blood 2008;112:4475-4484.

18 Fiddler CA, Parfrey H, Cowburn AS, Luo D, Nash GB, Murphy G, Chilvers ER: The Aminopeptidase CD13 Induces Homotypic Aggregation in Neutrophils and Impairs Collagen Invasion. PLoS One 2016;11:e0160108.

19 Pande A, Dorwal P, Jain D, Tyagi N, Mehra S, Sachdev R, Raina V: Expression of CD71 by flow cytometry in acute leukemias: More often seen in acute myeloid leukemia. Indian J Pathol Microbiol 2016;59:310-313.

20 Obeidi N, Pourfathollah AA, Soleimani M, Nikougoftar Zarif M, Kouhkan F: The Effect of Mir-451 Upregulation on Erythroid Lineage Differentiation of Murine Embryonic Stem Cells. Cell J 2016;18:165178.

21 Saunders AE JP: Modulation of immune cell signalling by the leukocyte common tyrosine phosphatase, CD45. Cell Signal 2010;22:339-348.

22 Kang R, Zhou Y, Tan S, Zhou G, Aagaard L, Xie L, Bunger C, Bolund L, Luo Y: Mesenchymal stem cells derived from human induced pluripotent stem cells retain adequate osteogenicity and chondrogenicity but less adipogenicity. Stem Cell Res Ther 2015;6:144.

-23 Yin M, Yuan Y, Cui Y, Hong X, Luo H, Hu X, Tang M, Hescheler J, Xi J: Puerarin Suppresses the Self-Renewal of Murine Embryonic Stem Cells by Inhibition of REST-MiR-21 Regulatory Pathway. Cell Physiol Biochem 2015;37:527-536.

24 Takahashi K, Tanabe K, Ohnuki M, Narita M, Ichisaka T, Tomoda K, Yamanaka S: Induction of pluripotent stem cells from adult human fibroblasts by defined factors. Cell 2007;131:861-872.

25 Liu T, Zhang S, Chen X, Li G, Wang Y: Hepatic differentiation of mouse embryonic stem cells in threedimensional polymer scaffolds. Tissue Eng Part A 2010;16:1115-1122.

-26 Gossmann M, Frotscher R, Linder P, Neumann S, Bayer R, Epple M, Staat M, Artmann AT, Artmann GM: Mechano-Pharmacological Characterization of Cardiomyocytes Derived from Human Induced Pluripotent Stem Cells. Cell Physiol Biochem 2016;38:1182-1198.

27 Li W, Yamashita H, Hattori F, Chen H, Tohyama S, Satoh Y, Sasaki E, Yuasa S, Makino S, Sano M, Fukuda K: Simple autogeneic feeder cell preparation for pluripotent stem cells. Stem Cell Res 2011;6:83-89.

28 Lowry WE, Richter L, Yachechko R, Pyle AD, Tchieu J, Sridharan R, Clark AT, Plath K: Generation of human induced pluripotent stem cells from dermal fibroblasts. Proc Natl Acad Sci USA 2008;105:2883-2888.

29 Jaenisch R, Young R: Stem cells, the molecular circuitry of pluripotency and nuclear reprogramming. Cell 2008;132:567-582.

30 Lin W, Modiano JF, Ito D: Stage-specific embryonic antigen (SSEA): determining the expression in canine glioblastoma, melanoma, and mammary cancer cells. J Vet Sci, in press. 


\section{Cellular Physiology Cell Physiol Biochem 2017;41:1661-1674

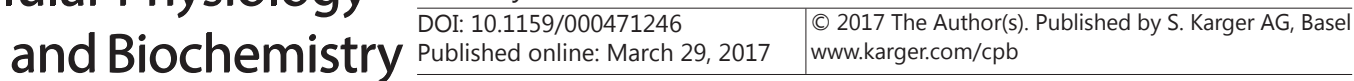 \\ Zhu et al.: AML and iPSCs}

-31 Ahmadian Baghbaderani B, Tian X, Scotty Cadet J, Shah K, Walde A, Tran H, Kovarcik DP, Clarke D, Fellner T: A Newly Defined and Xeno-Free Culture Medium Supports Every-Other-Day Medium Replacement in the Generation and Long-Term Cultivation of Human Pluripotent Stem Cells. PLoS One 2016;11:e0161229.

32 Zhou P, Wu G, Zhang P, Xu R, Ge J, Fu Y, Zhang Y, Du Y, Ye J, Cheng J, Jiang H: SATB2-Nanog axis links age-related intrinsic changes of mesenchymal stem cells from craniofacial bone. Aging (Albany NY) 2016;8:2006-2011.

-33 Fukuda T, Tani T, Haraguchi S, Donai K, Nakajima N, Uenishi H, Eitsuka T, Miyagawa M, Song S, Onuma M, Hoshino Y, Sato E, Honda A: Expression of Six Proteins Causes Reprogramming of Porcine Fibroblasts Into Induced Pluripotent Stem Cells With Both Active X Chromosomes. J Cell Biochem 2017;118:537-553.

-34 Shyh-Chang N, Daley GQ: Lin28: primal regulator of growth and metabolism in stem cells. Cell Stem Cell 2013;12:395-406.

35 Bianco C, Rangel MC, Castro NP, Nagaoka T, Rollman K, Gonzales M, Salomon DS: Role of Cripto-1 in stem cell maintenance and malignant progression. Am J Pathol 2010;177:532-540.

36 Liao J, Karnik R, Gu H, Ziller MJ, Clement K, Tsankov AM, Akopian V, Gifford CA, Donaghey J, Galonska C, Pop R, Reyon D, Tsai SQ, Mallard W, Joung JK, Rinn JL, Gnirke A, Meissner A: Targeted disruption of DNMT1, DNMT3A and DNMT3B in human embryonic stem cells. Nat Genet 2015;47:469-478.

-37 Shabestarian H, Ghodsi M, Mallak AJ, Jafarian AH, Montazer M, Forghanifard MM: DPPA2 Protein Expression is Associated with Gastric Cancer Metastasis. Asian Pac J Cancer Prev 2015;16:8461-8465.

-38 Oliviero G, Munawar N, Watson A, Streubel G, Manning G, Bardwell V, Bracken AP, Cagney G: The variant Polycomb Repressor Complex 1 component PCGF1 interacts with a pluripotency sub-network that includes DPPA4, a regulator of embryogenesis. Sci Rep 2015;5:18388.

-39 Thier M, Worsdorfer P, Lakes YB, Gorris R, Herms S, Opitz T, Seiferling D, Quandel T, Hoffmann P, Nothen MM, Brustle O, Edenhofer F: Direct conversion of fibroblasts into stably expandable neural stem cells. Cell Stem Cell 2012;10:473-479. 